\title{
PERIODIC POINTS AND TOPOLOGICAL ENTROPY OF MAPS OF THE CIRCLE
}

\author{
CHRIS BERNHARDT
}

\begin{abstract}
Let $f$ be a continuous map from the circle to itself, let $P(f)$ denote the set of integers $n$ for which $f$ has a periodic point of period $n$. In this paper it is shown that the two smallest numbers in $P(f)$ are either coprime or one is twice the other.
\end{abstract}

1. Introduction. Let $f$ be a continuous map of the circle into itself, let $P(f)$ denote the set of positive integers $n$ such that $f$ has a periodic point of (least) period $n$. If $P(f)$ does not consist of a single point, let $p_{1}$ and $p_{2}$ denote, respectively, the smallest and second smallest elements of $P(f)$. It will be shown that either $p_{1}$ and $p_{2}$ are coprime or $p_{2}=2 p_{1}$.

This result can then be combined with results in $[1,3$ and 6] to prove

TheOREM 1. Let $f \in C^{0}\left(S^{1}, S^{1}\right)$. Suppose that $P(f)$ contains more than one element. Let $p_{1}$ and $p_{2}$ denote the smallest elements of $P(f)$, with $p_{1}<p_{2}$.

If $2 p_{1} \neq p_{2}$ then:

(1) $p_{1}$ and $p_{2}$ are coprime;

(2) $\alpha p_{1}+\beta p_{2} \in P(f)$ where $\alpha$ and $\beta$ are any positive integers;

(3) The topological entropy of $f, h(f) \geqslant \log \mu_{p_{1} . p_{2}}$, where $\mu_{p_{1} . p_{2}}$ is the largest zero of $x^{p_{1}+p_{2}}-x^{p_{2}}-x^{p_{1}}-1$.

(4) There exists a map $f_{p_{1}, p_{2}} \in C^{0}\left(S^{1}, S^{1}\right)$ such that

$$
P\left(f_{p_{1}, p_{2}}\right)=\left\{\alpha p_{1}+\beta p_{2} \mid \alpha \in N^{+}, \beta \in N^{+}\right\} \cup\left\{p_{1}, p_{2}\right\}
$$

and $h\left(f_{p_{1}, p_{2}}\right)=\log \mu_{p_{1}, p_{2}}$

If $2 p_{1}=p_{2}$ there exists a map, $f_{p_{1}, p_{2}}$, with $P\left(f_{p_{1}, p_{2}}\right)=\left\{p_{1}, p_{2}\right\}$ and $h\left(f_{p_{1}, p_{2}}\right)=0$.

2. In this section the following theorem is proved.

Theorem 2.1. Let $f \in C^{0}\left(S^{1}, S^{1}\right)$. Suppose that $P(f)$ is not a singleton. Let $p_{1}, p_{2}$ denote the two smallest elements of $P(f)$. Then either $p_{1}$ and $p_{2}$ are coprime or $p_{2}=2 p_{1}$.

The theorem is trivially true if $p_{1}=1$, so throughout this section it will be assumed that $f$ has no fixed points.

Definition 2.2. Let $f$ be an endomorphism of the circle of degree 1 and let $F$ be a lifting of $f$. The rotation number $\rho(F, x)$ is defined by $\rho(F, x)=$ $\lim \sup _{n \rightarrow \infty}(1 / n)\left(F^{n}(x)-x\right)$, and the rotation set $\rho(F)=\{\rho(F, x): x \in \mathbf{R}\}$.

Received by the editors March 8, 1982 and, in revised form. June 14, 1982.

1980 Mathematics Subject Classification. Primary 58F20. 
The rotation set $\rho(F)$ is a closed interval or a single point, and a different lifting of $f$ just translates the rotation set by an integer (see [ 7 and 4 or 8]).

In [4 and 8] the following is shown.

Lemma 2.3. Let $f \in C^{0}\left(S^{1}, S^{1}\right)$ be a degree one map with rotation interval $[a, b]$. Then for any rational number $m / n \in[a, b]$, with $m$ and $n$ coprime, $n$ belongs to $P(f)$.

LEMMA 2.4. Let $a / b, c / d$ be two rational numbers contained in the interval $[0,1]$. Suppose that $a / b<c / d$ and that $b$ and $d$ have $a$ common factor. Then there exists $a$ rational number $m / n$ satisfying $a / b \leqslant m / n \leqslant c / d$, such that:

(i) $n<\max (b, d)$;

(ii) $n \notin\{b, d\}$.

Proof. The proof will be divided into two cases depending on whether the fractions $a / b, c / d$ are expressed in lowest terms or not.

Case 1. Suppose that both $a / b$ and $c / d$ are already in lowest terms, i.e. the numerator and denominator are coprime. Then both $a / b$ and $c / d$ will occur in the $\max (b, d)$ row of the Farey series. By elementary number theory there exists a rational number $m / n$, with required properties (see, for example, [5]).

Case 2. Suppose that $a / b$ and $c / d$ are not already in lowest terms. Cancellation either gives the required result immediately or reduces to the first case.

Proof of TheOREM 2.1. Since $f$ has no fixed points it must have degree one. Thus the rotation set is defined and, without loss of generality, may be assumed to be contained in the unit interval $[0,1]$.

Choose $x \in S^{1}$ such that $f^{p_{1}}(x)=x$ and choose $y \in S^{1}$ such that $f^{p_{2}}(y)=y$.

Suppose that $p_{1}$ and $p_{2}$ have a common factor. Then write $p_{1}=k q$ and $p_{2}=l q$ where $k$ and $l$ are coprime.

Let $\rho(x)=a / k q$ and $\rho(y)=b / l q$. Clearly $(a, k q)=1$, otherwise Lemma 2.3 would imply the existence of a periodic point with period smaller than $p_{1}$.

Suppose that $a / k q \neq b / l q$. Then applying Lemma 2.4 and then Lemma 2.3 shows that there exists a point of period $n$, where $n \neq p_{1}$ and $n<p_{2}$. This contradicts the definition of $p_{1}$ and $p_{2}$.

Thus $a / k q=b / l q$ and so $b k=a l$. Since $(k, l)=1, k$ divides $a$; but $(a, k q)=1$ and so $k=1$.

It has been shown that if $p_{1}$ and $p_{2}$ are not coprime then $p_{2}=l p_{1}$ and $\rho(x)=\rho(y)$.

Now consider the map $f^{p_{1}}$. This has a fixed point $x$, and $y$ is a point of period $l$. Clearly 1 and $l$ are the two smallest elements of $P\left(f^{p}\right)$. Since $f^{p}$ is of degree one there exists a lifting $g$ such that $\rho(x)=\rho(y)=0$.

Thus $g \in C^{0}(\mathbf{R}, \mathbf{R})$ and 1 and $l$ are the two smallest elements of $P(g)$, (if a lifting of a degree one map has a periodic point of period $k$, then so does the map). Sarkovskii's theorem then shows that $l=2$.

3. Proof of Theorem 1. Louis Block has extensively studied the case when $p_{1}=1$. When $p_{1}=1$, Theorem 1 is weaker then the results in [ 2 and 3].

Ito [6] has shown the following: 
Theorem 3.1. Let $f \in C^{0}\left(S^{1}, S^{1}\right)$. Let $m, n \in P(f)$ such that $m \geqslant 2, n \geqslant 2$ and $(m, n)=1$. Then $h(f) \geqslant \log \mu_{m, n}$ where $\mu_{m, n}$ is the largest zero of $x^{m+n}-x^{m}-x^{n}$ -1 .

In [1] the following is proved.

THEOREM 3.2. Let $f \in C^{0}\left(S^{1}, S^{1}\right)$. Let $p_{1}, p_{2}$ be the two smallest elements of $P(f)$. If $\left(p_{1}, p_{2}\right)=1$, then $\alpha p_{1}+\beta p_{2} \in P(f)$ for any positive integers $\alpha$ and $\beta$.

Thus statements (1), (2), and (3) of Theorem 1 are true.

To complete the proof it is only necessary to construct maps $f_{p_{1}, p_{2}}$, with minimal entropy and with minimal number of periodic points.

When $\left(p_{1}, p_{2}\right)=1$, Ito [6] constructs a map $f_{p_{1}, p_{2}}: S^{1} \rightarrow S^{1}$. By looking at the associated $A$-graph he shows that $h\left(f_{p_{1}, p_{2}}\right)=\log \mu_{p_{1}, p_{2}}$. The $A$-graph also shows that $P\left(f_{p_{1}, p_{2}}\right)=\left\{\alpha p_{1}+\beta p_{2} \mid \alpha \in \mathbf{N}^{+}, \beta \in \mathbf{N}^{+}\right\} \cup\left\{p_{1}, p_{2}\right\}$.

Now consider the case $p_{2}=2 p_{1}$. Let $S^{1}=\mathbf{R} / \mathbf{Z}$ and let $f_{1.2}: S^{1} \rightarrow S^{1}$ be the map induced from $F_{1,2}: \mathbf{R} \rightarrow \mathbf{R}$ defined by

$$
F_{1,2}(x)= \begin{cases}2 x, & 0 \leqslant x \leqslant \frac{1}{3}, \\ -x+1, & \frac{1}{3} \leqslant x \leqslant \frac{2}{3}, \\ 2 x-1, & \frac{2}{3} \leqslant x \leqslant 1,\end{cases}
$$

and $F(x+k)=F(x)$ for $k \in \mathbf{Z}$.

It is easily checked that $f_{1,2}$ has only periodic points of periods 1 and 2 .

Similarly, let $f_{p .2 p}: S^{1} \rightarrow S^{1}$ be the map induced from $F_{p .2 p}: \mathbf{R} \rightarrow \mathbf{R}$ defined by

$$
F_{p, 2 p}(x)= \begin{cases}2 x+1 / p, & 0 \leqslant x \leqslant 1 / 3 p, \\ -x+2 / p, & 1 / 3 p \leqslant x \leqslant 2 / 3 p, \\ 2 x, & 2 / 3 p \leqslant x \leqslant 1 / p, \\ x+1 / p, & 1 / p \leqslant x \leqslant 1,\end{cases}
$$

and $F_{p .2 p}(x+k)=F_{p .2 p}(x)$ for $k \in \mathbf{Z}$. This map has only periodic points of period $p$ and $2 p$. Clearly $h\left(f_{p .2 p}\right)=0$.

\section{REFERENCES}

1. C. Bernhardt, Periodic orbits of continuous mappings of the circle without fixed points, Ergodic Theory and Dynamical Systems 1 (1981), 413-417.

2. L. Block, Periods of periodic points of maps of the circle which have a fixed point. Proc. Amer. Math. Soc. 82 (1981), $481-486$.

3. L. Block, E. M. Coven and Z. Nitecki, Minimizing topological entropy for maps of the circle. Ergodic Theory and Dynamical Systems 1 (1981), 145-149.

4. L. Block, J. Guckenheimer, M. Misurewicz and L. Young, Periodic points and topological entropy of one dimensional maps, Global Theory of Dynamical Systems, Lecture Notes in Math., vol. 819. Springer-Verlag, Berlin and New York, 1980.

5. C. Hardy and E. Wright, An introduction to the theory of numbers, Clarendon Press, Oxford, 1954, p. 23.

6. R. Ito, Minimal entropy for endomorphisms of the circle, preprint, Waseda University, Tokyo, Japan.

7. Rotation sets are closed, Math. Proc. Cambridge Philos. Soc. 89 (1981), 107-111.

8. S. Newhouse, J. Palis and F. Takens, Stable families of dynamical sy'stems I: Diffeomorphisms, Inst. Hautes Etudes Sci. Publ. Math. (to appear).

Department of Mathematics, Southern Illinois University, Carbondale, Illinois 62901

Current address: Department of Mathematics, Lafayette College, Easton, Pennsylvania 18042 